\title{
Mitochondrial dysfunction and delayed hepatotoxicity: another lesson from troglitazone
}

\author{
N. L. Julie • I. M. Julie • A. I. Kende • G. L. Wilson
}

Received: 3 March 2008 / Accepted: 26 June 2008 / Published online: 23 August 2008

(C) Springer-Verlag 2008

\begin{abstract}
Aims/hypothesis Troglitazone was approved for treatment of type 2 diabetes mellitus, but by 2000 it had been removed from all world markets due to severe drug-induced liver injury. Even today, we still do not know how many patients sustained a long-term liver injury. No system is in place to acquire that knowledge. Regarding toxicity mechanisms, controversy persists as to which ones are class effects of thiazolidinediones (TZDs) and which are unique to troglitazone. This study aims to provide long-term outcome data and new insights on mechanisms of troglitazone-induced liver injury.

Methods This case series reports the liver injuries sustained by eleven type 2 diabetic patients treated with troglitazone between 1997 and 2000. Exhaustive review of medical records was performed for all patients. Long-term outcomes
\end{abstract}

Electronic supplementary material The online version of this article (doi:10.1007/s00125-008-1133-6) contains supplementary material, which is available to authorised users.

N. L. Julie $\cdot$ A. I. Kende

Department of Pathology,

Shady Grove Adventist Hospital,

Rockville, MD, USA

I. M. Julie

Washington University School of Medicine,

St Louis, MO, USA

G. L. Wilson

Department of Cell Biology,

University of South Alabama, College of Medicine,

Mobile, AL, USA

N. L. Julie $(\bowtie) \cdot$ I. M. Julie

7609 Exeter Road,

Bethesda 20814 MD, USA

e-mail: neilljulie@aol.com were available for all the non-fatal cases. A comprehensive literature review was also performed.

Results Long-term liver injury progressing to cirrhosis was identified in seven patients. All eleven cases had liver injury patterns consistent with troglitazone toxicity. Analysis of these cases and of the experimental troglitazone toxicity data points to mitochondrial toxicity as a central factor. The general clinical patterns of mitochondrial hepatotoxic events are reviewed, as are the implications for other members of the TZD family.

Conclusions/interpretation This analysis enables the liver injury induced by troglitazone to be better understood. In future cases of delayed drug-induced liver injury that progresses after discontinuation, the possibility of mitochondrial toxicity should be considered. When appropriate, this can then be evaluated experimentally. Such proactive investigation may anticipate clinical risk before a large-scale therapeutic misadventure occurs. Drug-induced liver injury due to mitochondrial hepatotoxins may be less unpredictable than has previously been surmised.

Keywords Apoptosis · Cirrhosis · Drug-induced liver injury · Hepatotoxicity · Liver · Long-term · Mechanism . Mitochondria $\cdot$ Thiazolidinedione $\cdot$ Troglitazone

\begin{tabular}{ll}
\multicolumn{2}{l}{ Abbreviations } \\
ALT & alanine aminotransferase \\
AST & aspartate aminotransferase \\
CK-18 & cytokeratin-18 \\
NAFLD & non-alcoholic fatty liver disease \\
NASH & non-alcoholic steatohepatitis \\
OXPHOS & oxidative phosphorylation \\
PPAR & peroxisome proliferator-activated receptor \\
ROS & reactive oxygen species \\
TZD & thiazolidinedione
\end{tabular}


In the long-standing tradition of medical education, we will make frequent use of case studies, one of the most effective ways to render complex issues both accessible and engaging.

Jerry Avorn (2004) Powerful Medicines. New York, Vintage Books, p 19

\section{Introduction}

Troglitazone was prescribed for approximately 2,000,000 patients in the USA, UK (only from October to December, 1997) and Japan, yet became the second most common cause of fatality reported for drug-induced acute liver failure worldwide between 1970 and 2004, surpassed only by acetaminophen [1]. Despite this, the full extent and frequency of troglitazone-related injury has not been quantified and longer term consequences of exposure have not been explored. Extended follow-up has not been arranged, funded or expedited. The medical community needs to better understand the full nature, impact and mechanisms of this exposure episode. Such an understanding would offer valuable insights on delayed drug-induced liver injury and also on the relative safety of pioglitazone, rosiglitazone and other related agents.

Identification of delayed hepatotoxicity in the diabetic population is especially difficult. The prevalence of nonalcoholic fatty liver disease (NAFLD) can complicate recognition of such injury. Moreover, diabetic patients and patients with insulin resistance may have a pre-existing defect in mitochondrial number or function $[2,3]$.

The role of mitochondrial dysfunction

The pathophysiology of delayed drug-induced liver injury is poorly understood. Historical labels such as 'idiosyncratic and intrinsic' are oversimplified [4] and sometimes unhelpful. The more recent substitution of 'unpredictable and predictable' does not improve matters, since unpredictability may simply represent a failure to identify the factors underlying the injury. In this paper we explore one such important and under-recognised underlying factor: the role of mitochondrial dysfunction/disruption.

Mitochondrial dysfunction and disruption is a major component in many liver diseases. It has been described in non-alcoholic steatohepatitis (NASH) [5, 6], alcoholic liver disease [7, 8] and Wilson's disease [9] to name just a few, but its role in drug-induced liver injury has generally been overlooked (except when accompanied by severe microvesicular steatosis). A recent retrospective study of druginduced liver fatalities was performed using the WHO database [1]. Of the ten most common drugs associated with drug-induced liver fatality, mitochondrial dysfunction/disruption is a major component in at least five of them. Furthermore, mitochondrial dysfunction plays a key role in non-hepatic drug toxicities such as cardiomyopathy and rhabdomyolysis [10-13].

Troglitazone is an instructive example of a drug whose toxicity in the type 2 diabetic population appears to primarily involve mitochondrial dysfunction/disruption. This inferred conclusion will be based on an analysis of this case series and is also powerfully supported by cellular in-vitro studies and animal experiments [14-17]. The opposite premise, taken by some, which claims that this animal and cellular data are not relevant to the human experience, is far harder to defend.

The cases presented here demonstrate a spectrum of injury. The first four were patients treated by one of the authors, the other seven were analysed as part of the expert review process for legal proceedings. The pathology reports and not the slides were reviewed in the seven expert-review cases.

\section{Methods}

\section{Patient case reports}

This is a case series report of 11 patients. Patients 1 to 4 were patients treated by one of the authors (N. L. Julie). The next seven (patients 5-11) had their medical records exhaustively and completely reviewed. Full histories for all patients are in the Electronic supplementary material (ESM). In all cases, the stated age is age at the time troglitazone treatment was started.

Patient 1. The patient was a 65-year-old woman (Table 1) with mixed hepatocellular-cholestatic jaundice after 6 weeks of troglitazone $400 \mathrm{mg} /$ day. All other alternative causes for her hepatitis were methodically excluded. A liver biopsy revealed bridging necrosis and fibrosis (Table 2). Within several months of discontinuation, all of her liver enzymes had returned to normal except for a mildly elevated gamma glutamyl transpeptidase.

The patient was lost to follow-up for 6 years. Then, 9 years after troglitazone discontinuation, she presented with cholestasis, possibly precipitated by ceftriaxone, lisinopril or sepsis. After treatment and change of those medications her liver enzymes slowly improved. She is now once again asymptomatic [18].

Patient 2. This was a 73-year-old woman who had a 27year history of type 2 diabetes. She was on troglitazone for 4 months. The drug was stopped because of markedly abnormal liver enzymes. 
Table 1 Summary of patients suffering liver injury associated with troglitazone therapy

\begin{tabular}{|c|c|c|c|c|c|c|c|c|}
\hline \multirow[t]{2}{*}{$\begin{array}{l}\text { Patient } \\
\text { number }\end{array}$} & \multirow[t]{2}{*}{ Sex } & \multirow[t]{2}{*}{$\begin{array}{l}\text { BMI } \\
\left(\mathrm{kg} / \mathrm{m}^{2}\right)\end{array}$} & \multirow[t]{2}{*}{$\begin{array}{l}\text { Duration of } \\
\text { diabetes at therapy } \\
\text { start (years) }\end{array}$} & \multirow[t]{2}{*}{$\begin{array}{l}\text { Age at start } \\
\text { (years) }\end{array}$} & \multirow[t]{2}{*}{$\begin{array}{l}\text { Time on TZ } \\
\text { (months) }\end{array}$} & \multicolumn{2}{|c|}{$\begin{array}{l}\text { Duration to clinically } \\
\text { apparent liver injury } \\
\text { (months }\end{array}$} & \multirow[t]{2}{*}{ Age at death } \\
\hline & & & & & & On drug & Off drug & \\
\hline 1 & $\mathrm{~F}$ & 61.3 & 4 & 65 & 1.5 & 1.5 & NA & - \\
\hline 2 & $\mathrm{~F}$ & 25.0 & 27 & 73 & 4 & 4 & NA & - \\
\hline 3 & $\mathrm{~F}$ & 22.5 & 7 & 68 & 7 & 7 & NA & 69 \\
\hline 4 & $\mathrm{~F}$ & 26.6 & 8 & 53 & 7 & 7 & NA & - \\
\hline 5 & M & 44.3 & 8 & 44 & 21 & NA & 2 & 50 \\
\hline 6 & M & Unknown & 5 & 65 & 35 & NA & 1.5 & 71 \\
\hline 7 & $\mathrm{~F}$ & 28.2 & 2 & 49 & 4.5 & 4.5 & NA & - \\
\hline 8 & $\mathrm{~F}$ & 23.2 & 7 & 69 & 6 & 6 & NA & - \\
\hline 9 & M & 31.1 & 4 & 61 & 3.5 & NA & 3 & - \\
\hline 10 & $\mathrm{~F}$ & Unknown & 6 & 59 & 15 & NA & 3 & Status unknown \\
\hline 11 & $\mathrm{~F}$ & 25.8 & 5 & 66 & 10 & NA & 7 & Status unknown \\
\hline Mean \pm SD & & $39.1 \pm 12.7$ & $8 \pm 7$ & $61 \pm 9$ & $10.4 \pm 9.9$ & $5 \pm 2$ & $3.3 \pm 2.2$ & $63 \pm 12$ \\
\hline
\end{tabular}

Patients 1 to 4 were personal patients treated by one of the authors (N. L. Julie)

F, female; M, male; NA, not applicable

Within a month of discontinuation, the patient developed chronic encephalopathy, which slowly improved but never completely resolved. She had her first liver biopsy 5 years after troglitazone discontinuation, which showed cirrhosis consistent with prior drug toxicity (Fig. 1a,b). She currently resides in a nursing home, disabled by her chronic encephalopathy.
Patient 3. This patient was a 68 -year-old woman with a 7year history of type 2 diabetes. She was on troglitazone for approximately 7 months. Treatment was discontinued because of fatigue, confusion and jaundice. A biopsy (Table 2) showed cirrhosis and panlobular collapse. Despite medical support, she died while awaiting liver transplantation [19].

Table 2 Liver pathology

\begin{tabular}{|c|c|c|c|c|c|c|c|c|}
\hline $\begin{array}{l}\text { Patient } \\
\text { number }\end{array}$ & $\begin{array}{l}\text { Months from } \\
\text { drug start to } \\
\text { biopsy }\end{array}$ & $\begin{array}{l}\text { Bridging } \\
\text { fibrosis and/ } \\
\text { or cirrhosis }\end{array}$ & $\begin{array}{l}\text { Portal } \\
\text { inflammation }\end{array}$ & $\begin{array}{l}\text { Microvesicular } \\
\text { steatosis }^{\mathrm{a}}\end{array}$ & $\begin{array}{l}\text { Macrovesicular } \\
\text { steatosis }^{\mathrm{a}}\end{array}$ & $\begin{array}{l}\text { Broad bands } \\
\text { or pan-lobular } \\
\text { collapse }\end{array}$ & $\begin{array}{l}\text { Bile ductule } \\
\text { proliferation }\end{array}$ & $\begin{array}{l}\text { Comments } \\
\text { on eosinophils }\end{array}$ \\
\hline 1 & 2 & Yes & Yes & N.N. & 0 & No & N.N. & Numerous \\
\hline 2 & 60 & Yes, early C & Mild & 0 & 0 & No & N.N. & Rare \\
\hline 3 & 7 & $\mathrm{C}$ & N.N. & N.N. & N.N. & Yes & Yes & N.N. \\
\hline $4 a$ & 16 & Yes & Yes & N.N. & 0 & No & N.N. & Moderate \\
\hline $4 b$ & 29 & Yes & No & N.N. & 0 & No & N.N. & N.N. \\
\hline $4 c$ & 88 & Gone & No & N.N. & 0 & No & Yes & N.N. \\
\hline 5 & 47 & Yes, C & Yes & N.N. & 0 & Yes & Yes & N.N. \\
\hline $6 a$ & 36 & $\mathrm{C}$ & Yes & + & + & No & No & No \\
\hline $6 b$ & 44 & $\mathrm{C}$ & No & + & + & No & No & No \\
\hline $7 \mathrm{a}$ & 4 & Yes & Yes & N.N. & N.N & Yes & N.N. & N.N. \\
\hline $7 b$ & 10 & New $\mathrm{C}$ & Yes & + & N.N. & Yes & N.N. & N.N. \\
\hline 8 & 5 & Yes vs early $\mathrm{C}$ & Yes & ++ & N.N. & N.N. & N.N. & N.N \\
\hline $9 a$ & 20 & $\mathrm{C}$ & Yes & N.N. & N.N. & No & No & N.N. \\
\hline $9 b$ & 24 & $\mathrm{C}$ & Yes & N.N. & + & No & No & No \\
\hline 10 & 30 & $\mathrm{C}$ & Mild & N.N. & 0 & Yes & Yes & No \\
\hline 11 & 22 & Yes & N.N. & N.N. & + & Yes & Yes & No \\
\hline Total $(n)$ & & $11 / 11$ patients & 9/11 patients & 3/11 patients & 4/11 patients & $5 / 11$ patients & $5 / 11$ patients & 3/11 patients \\
\hline
\end{tabular}

Patients 1 to 4 were personal patients treated by one of the authors (N. L. Julie)

${ }^{\mathrm{a}}$ Micro- and macrovesicular steatosis was defined as follows: $0=$ none, $+=$ mild, $++=$ moderate or prominent

C, cirrhosis; N.N., not noted 


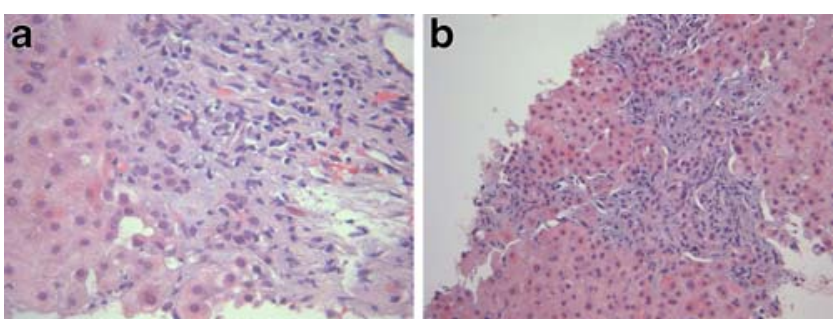

Fig. 1 Biopsy from patient 2 in 2005. a Ductular proliferation (centre) with mild portal inflammation (haematoxylin and eosin). Magnification $\times 400$. b Bridging fibrosis with ductular proliferation (haematoxylin and eosin). Magnification $\times 200$

Patient 4. This 53-year-old woman had an 8-year history of type 2 diabetes. She was on troglitazone for 7 months. Within 9 days of discontinuation, the patient developed a bilirubin level of $110.5 \mathrm{mmol} / \mathrm{l}$; alanine aminotransferase (ALT) and aspartate aminotransferase (AST) were 15 times the upper limits of normal (i.e. ALT, $36 \mathrm{U} / \mathrm{l}$; AST, $35 \mathrm{U} / 1)$. She subsequently had three liver biopsies over 4 years. The first biopsy (1998) was done 10 months after troglitazone discontinuation and showed chronic active hepatitis with bridging fibrosis (troglitazone-induced hepatotoxicity) (Fig. 2a-d). The second biopsy in October, 1999 was 'highly fragmented and suggests nodule formation' with essentially no inflammation. The third biopsy in 2004 showed diminishment of fibrosis. There was no fragmentation in the third biopsy, as had been seen previously (Fig. 2e, f). Since 2007 she has been asymptomatic.

Patient 5. This male patient was 44 years old when started on troglitazone for 21 months. Within 1 year of discontinuation the patient developed signs of steadily progressive portal hypertension. He died from complications of his liver disease 3 years after drug cessation.

Patient 6. Aged 65 years at inception of troglitazone therapy, this male patient took the drug for 35 months. Within 6 weeks of drug discontinuation, the patient had developed ascites. Biopsy confirmed cirrhosis and both micro- and macrovesicular steatosis. This progressed, and the patient developed encephalopathy and eventual hepatorenal syndrome. He died 3 years after drug discontinuation.

Patient 7. This 49-year-old woman was on troglitazone for 4.5 months. She had two liver biopsies. The first revealed submassive hepatic necrosis and the second showed microvesicular steatosis and progression to cirrhosis.
Patient 8. A 69-year-old woman, this patient started on troglitazone for 4 months. Treatment was stopped and restarted for an additional 2 months. She presented with acute liver failure, ascites and myositis. Liver biopsy showed moderate microvesicular steatosis. This was the only patient in whom Oil red 0 staining was done. She developed ascites and varices after her acute liver failure resolved. As of mid-2004, she was alive, but had persisting signs and symptoms of portal hypertension and cirrhosis.

Patient 9. This male patient was 61 years old when started on troglitazone for 3 months. By 1 year after discontinuation of troglitazone, the patient developed signs of portal hypertension and bleeding oesophageal varices. Biopsies showed microvesicular steatosis and prominent Mallory bodies (hyaline).
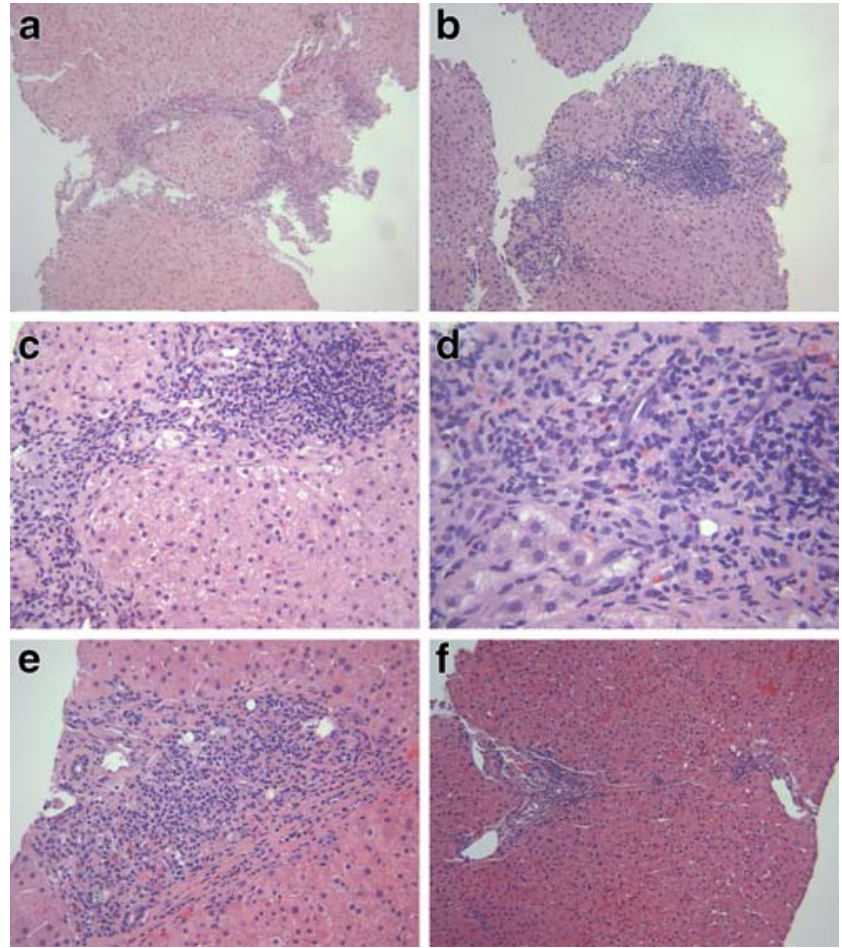

Fig. 2 a-d First biopsy (1998) and (e, f) third biopsy (2004) from patient 4. a Fragmentation with bridging fibrosis approaching cirrhosis (haematoxylin and eosin). Magnification $\times 100$. b Bridging fibrosis with significant portal inflammation (haematoxylin and eosin). Magnification $\times 100$. $\mathbf{c}$ Bridging fibrosis and portal inflammation with lymphocytes, pigmented macrophages and a few eosinophils (haematoxylin and eosin). Magnification $\times 200$. d Portal inflammation with lymphocytes and a few eosinophils (haematoxylin and eosin). Magnification $\times 400$. e Portal inflammation with lymphocytes, plasma cells, a few eosinophils and ductular proliferation (haematoxylin and eosin). Magnification $\times 200$. f Thin residual fibrous bridge without inflammation (haematoxylin and eosin). Magnification $\times 100$ 
Patient 10. Aged 59 years when started on troglitazone, this female patient took the drug for 15 months. Within 3 months of discontinuing the drug, she had developed ascites, thrombocytopenia and other signs of portal hypertension. The patient progressively worsened but was lost to follow-up after 2003.

Patient 11. A 66-year-old woman, this patient started on troglitazone for 10 months. Within 1 year of discontinuation, she presented with bleeding oesophageal varices, subsequently developing persistent symptoms of portal hypertension and hepatic hydrothorax. A biopsy confirmed cirrhosis.

\section{Results}

Summary of clinical results

This case series illustrates the heterogeneous pattern of drug-induced liver injury associated with troglitazone. The 11 cases above had clinical patterns that were either entirely consistent with troglitazone toxicity or highly inconsistent with progression of any identified or probably pre-existing liver condition. Progression to chronic injury is clearly seen in patients 2 and patients 6 to 11 . For all 11 patients, more detailed histories are provided in the ESM. With regard to troglitazone, the clinical features of this series are consistent with and suggestive of an injury that is mainly mitochondrial, as will be explained below.

The patterns of liver injury are pleiomorphic. Acute liver failure with submassive necrosis and death was seen in patient 3 . There was obvious acute liver injury with eventual cirrhosis in patients 2 and 7. Finally, in patients 6, 9, 10 and 11 , late detection of insidious cirrhosis, often presenting as portal hypertension, was seen.

In this series and in previously reported cases of drug-induced liver injury associated with troglitazone, the symptoms experienced were none [20], mild or non-specific. Temporal onset of symptoms of injury was delayed. In the published literature, the median time from onset to symptoms of acute liver failure is 4 to 5 months [21]. However, the range is tremendous, from a minimum of 4 days [22] to more than 2 years [23]. In this series, troglitazone-induced liver injury associated with acute hepatocellular or mixed injury occurred in this same time frame. However, cases presenting with cirrhosis or portal hypertension were diagnosed even later (1.5 to 3 years from start of exposure in patients 5, 6, 9, 10 and 11.).

Pathological investigation revealed several noteworthy features. Three of these cases had microvesicular steatosis (patients 6,7 and 8). Microvesicular steatosis was the central finding in one case [24]. Other cases showed cirrhosis, including one case with histological evidence of gradual regression (patient 4 ). In many of the cases, frequently seen features included: bile ductular proliferation or bile stasis/ cholestasis, and varying degrees of necrosis, inflammation and fibrosis. Progression of injury after drug discontinuation was a common occurrence in these patients and in previous case reports [19, 22, 25, 26].

Other mitochondrial hepatopathies are very similar. Symptoms are often non-specific and identical to those seen in the patients above. Onset is delayed. ALT and AST elevations may be absent, mild or moderate. Late histological findings include bile ductular proliferation, cholestasis and fibrosis [27-29]. Although microvesicular steatosis is looked for as a pathognomonic sign of this disorder, small vesicles can coalesce into macrovesicular fat [30]. Thus, this early lesion may disappear and furthermore can be overlooked with routine haematoxylin and eosin staining [31].

In particular, progression after discontinuation often occurs with mitochondrial hepatotoxins. This is generally not the case in drug-induced liver injury hepatitis that is predominantly immune-mediated. For example, druginduced hepatitis due to methyldopa and tienilic acid ceases with drug discontinuation [32].

\section{Discussion}

The clinical spectrum of liver injury in the group of patients discussed here is broad. Toxicity ranges from acute and severe to chronic and insidious. The implications of this study with regard to the other glitazones will be discussed in detail later on.

The breadth of troglitazone-induced injury seen in the patients analysed is common to several diseases and xenobiotics that disrupt mitochondrial function. Examples include valproic acid [33], amiodarone [34-36], Wilson's disease [8], Alpers' syndrome [37] and (to some extent) alcohol [7]. These too have similarly pleiomorphic potential, extending from acute hepatitis to cirrhosis. However, with regard to drug-induced liver injury, the current Adverse Event Reporting/Medwatch system in the USA captures much of the hepatitis and acute liver failure, but almost none of the cirrhosis and portal hypertension. Prior to 2007, the Medwatch reporting system in the USA precluded regulatory reviewers from getting patients names or directly contacting treaters for longer term follow-up of outcomes.

What is the reason for this broad spectrum of injury? This clinical pleiomorphism can be explained by and is a manifestation of the different general mitochondrial damage pathways. The resultant clinical cell-injury patterns can be summarised in four main categories.

1. Reactive metabolite/reactive oxygen species (ROS) formation and severe oxidative stress, which can arise 
due to drug-induced dysfunction of oxidative phosphorylation (OXPHOS) and impaired electron transport through the respiratory chain. Mitochondria are the primary source of ROS [11]. This can cause steatohepatitis or progression to cirrhosis. [38, 39].

2. Rapid and severe impairment of $\beta$-oxidation of fatty acids, which can give rise to microvesicular steatosis and ultimately result in acute Reye's syndrome type liver failure.

3. Induction of widespread cell death consisting of necrosis can predominate. The extreme form is massive or submassive hepatic necrosis due to generalised mitochondrial destruction/failure. This is probably the pattern seen in the clinical case reports of acute liver failure due to a new drug with mitochondrial toxicity

4. The initiation and steady progression of apoptosis or necroapoptosis. This is clinically a subtle form of liver injury. It easily goes undetected if the patient is asymptomatic. Newer, more sensitive tests for apoptosis such as caspase-3-generated cytokeratin-18 (CK-18) may help in its recognition [40].

With many of the toxins/diseases that adversely affect mitochondria, one of the above categories may predominate, but there is often substantial overlap. One category of injury may lead to another. The common theme is that these agents and disorders all converge on pathways that result in significant disruption of mitochondrial structure and/or function.

Since troglitazone was withdrawn, much has been learned about the potential mechanisms of its hepatotoxicity. There is rapidly accumulating experimental evidence confirming that mitochondrial dysfunction had a central role. Scatena noted that the evidence of a particular mitochondrial derangement induced by the so-called peroxisome proliferator-activated receptor (PPAR) ligands could explain some hideous aspects related to the pharmacotoxicological profile of these molecules' [41].

Excellent reviews on this topic have been published [16, 17, 42]. For example, Masubuchi [42] concluded that troglitazone itself, rather than its metabolites, was the main agent responsible for liver injury and that mitochondria are 'the major targets in the toxicity'. Feinstein proposed that thiazolidinediones (TZDs) interact directly with mitochondria, altering bioenergetics and potentially increasing ROS production and causing ATP depletion [17]. Evidence for PPAR $\gamma$ independent mitochondrial effects in the glitazone family can be seen from the fact that the rank order for efficacy to elicit certain key responses for TZDs is the inverse of the known $\operatorname{PPAR} \gamma$ receptor binding affinity (rosiglitazone>pioglitazone $>$ troglitazone). Moreover, PPAR $\gamma$ antagonists and transcriptional and translational inhibitors did not block these effects of TZDs. Thus, such responses as alteration of bioenergetics, metabolism and mitochondrial function are PPAR $\gamma$-independent [17]. The order of toxicity by which TZDs cause mitochondrial dysfunction is troglitazone>pioglitazone and rosiglitazone $[14,43]$. The hierarchy of clinical and cellular hepatotoxicity correlates strongly to mitochondrial impairment and not to PPAR $\gamma$ affinity $[14,17,43]$.

In human hepatocytes, troglitazone, but not pioglitazone, induced ROS production accompanied by drop in mitochondrial transmembrane potential and profound mitochondrial ultrastructural changes. These mitochondrial changes were inhibited by $N$-acetyl cysteine [15]. Furthermore, enhanced susceptibility to cell injury in conditions of preexisting oxidative stress/mitochondrial impairment has been demonstrated by Ong and Boelsterli in a mouse model of silent mitochondrial abnormality [44]. This is relevant to patients with pre-existing oxidative stress due to NAFLD or glutathione depletion.

Alternative or additional effects of troglitazone include inhibition of a key bile transporter, bile salt export protein $[45,46]$. This inhibition can therefore contribute substantially to the cholestasis that was often seen. Retention of bile salts can feed back, enhancing toxicity $[42,46]$.

\section{The TZD family-unique vs class effects}

What data are available on the other glitazones regarding hepatotoxicity, mitochondrial dysfunction/disruption and oxidative stress? There are few published cases of severe hepatotoxicity due to pioglitazone and rosiglitazone [47-52]. Thus, with those TZDs hepatocyte/mitochondrial injury rarely exceeds the threshold leading to clinically significant liver injury. Nonetheless, numerous experimental studies have shown mitochondrial impairment caused by TZDs that remain on the market. Rosiglitazone (and to a lesser degree, pioglitazone [53]) has been shown to inhibit OXPHOS complex I [13]. Rosiglitazone, like troglitazone, rapidly reduced mitochondrial transmembrane potential [14]. In an in-vivo diabetic mouse model, rosiglitazone and troglitazone induced microvesicular steatosis in the liver, an indicator of impairment of $\beta$-oxidation and mitochondrial function [54]. Significantly fewer experimental data are available for pioglitazone. However, a recent study showed high-affinity binding by pioglitazone to a mitochondrial protein named MitoNEET [55].

These clinical and experimental data support the conclusion that these rare pioglitazone- and rosiglitazonerelated hepatotoxic events also include cases of delayed drug-induced liver injury due to mitochondrial disruption/ dysfunction. Yet only troglitazone consistently crosses the threshold of inducing clinically obvious and significant hepatic damage in a substantial percentage of patients.

Other noteworthy points that differ among glitazones should be briefly mentioned. There are differences in reactive 
metabolites formed [56]. There are also differences in dose exposures, these being highest with troglitazone (clinical dose, rosiglitazone 4-8 $\mathrm{mg}$, pioglitazone $15-45 \mathrm{mg}$, troglitazone $200-600 \mathrm{mg}$ ), thus intensifying the toxic effects of troglitazone. Attributes unique to troglitazone include the presence of a pro-oxidant phenoxyl radical-forming chroman ring. Finally, inhibition of bile salt export protein has been demonstrated only with troglitazone [45].

What are the implications of the above? Certainly, careful testing for mitochondrial impact should be undertaken with unapproved or new glitazones or glitazars (e.g. muraglitazar). With regard to the TZDs currently in use, clinicians should be aware of this potential toxicity and should be vigilant in patients most at risk. Additionally, consideration of non-liver mitochondrial toxicities (i.e. rhabdomyolysis) should not be forgotten, especially when glitazones are co-administered with statins [13, 57, 58].

A key implication is that, given the right circumstances and a susceptible host, all TZDs have some capacity to trigger hepatocyte and other cell death pathways. These events may be mild or extreme and may occur after years on the drug. Frequency depends on the characteristics of the drug. Timing depends on situational covariables such as concurrent drugs or inflammation [59], cholestatic events, superimposed illnesses, degree of pre-existing mitochondrial impairment etc.

On the other hand, the currently approved and prescribed glitazones appear to have little effect in inducing ROS formation and oxidative stress. In fact, if they decrease OXPHOS, they may cause a countervailing drop in ROS that is partially protective until drug discontinuation. PPAR $\gamma$ ligands also have anti-inflammatory effects. They therefore should have a lower capacity than troglitazone to induce liver fibrosis/cirrhosis and may actually benefit some patients with NASH.

However, uncertainties about the hepatotoxicity of the current TZDs remain. Thus clinicians should initiate a thorough hepatology work-up when a patient on a TZD develops unexpected signs of severe hepatic dysfunction/ decompensation or portal hypertension.

Regrettably, no surveillance system has yet been implemented to determine the long-term liver injuries caused by troglitazone, thus leaving many concerns unresolved.

\section{Conclusions}

One can reasonably infer from this clinical series and the scientific experimental evidence that mitochondrial dysfunction plays a central role in troglitazone hepatotoxicity. A generalised awareness among physicians of this type of drug-induced injury mechanism and its components (ROS, mitochondrial permeability transition, apoptosis and massive necrosis) would provide a clinically useful frame of reference for evaluating other drugs. This would enable more accurate appraisal of late and long-term drug-induced liver injury by clinicians and toxicologists.

These reported cases provide insights into the presentation, management approach and diagnostic work-up of clinically similar patients. Such knowledge can be applied to other potential hepatotoxins acting via mitochondrial pathways. With this type of hepatotoxicity, the symptoms at presentation are pleiomorphic. Patients present with non-specific abdominal complaints, fatigue and progressive signs of hepatic dysfunction that occur weeks to months after initiation of therapy. These symptoms are not necessarily reflected in a marked elevation of AST or ALT. The injury will often progress or persist for months after drug discontinuation. If pathways of oxidative stress or steatohepatitis predominate, then significant fibrosis and cirrhosis may result.

This paper's analysis raises important questions for the TZD family and those members currently on the market. It is likely that toxic mitochondrial effects contributed to the clinical demise of ciglitazone, englitazone and possibly muraglitazar. However, clinically significant hepatotoxic events should be very rare with the TZDs still on the market. Furthermore, pioglitazone and rosiglitazone probably produce significantly less ROS than troglitazone and should therefore cause less fibrogenic liver injuries.

What action should clinicians take when faced with liver enzyme elevation in patients on a TZD? In outpatients on a TZD with unexplained tripling of AST or ALT from baseline, a thorough drug and acetaminophen history should be obtained. Other causes (i.e. viral, gallstones etc.) should be excluded. The TZD can be stopped pending these results. In more complicated or hospitalised patients, the TZD and other potentially hepatotoxic medications should be immediately discontinued. Alternative diagnoses should be ruled out and signs of portal hypertension, cirrhosis or significant hepatic dysfunction should prompt involvement of a hepatology consultation and consideration of liver biopsy. In both groups, clinicians should be alert to the occurrence of drug-induced liver injury superimposed upon a pre-existing liver condition. Chronic hepatitis $\mathrm{C}$ or B virus infection, alcohol abuse or NAFLD may be present. However, as has been seen with highly active antiviral therapy for HIV, the hepatotoxicity of the drug appears to be augmented by the pre-existing hepatic infection/disorder [60-63].

Unfortunately, very few tests are currently available for the identification of mitochondrially mediated hepatotoxicity. Lactic acid elevation is generally known to be a very insensitive marker. Concurrent elevation of creatine kinase is helpful when present [57]. It connotes mitochondrial damage affecting multiple target organs. When liver biopsies are performed, tissue should be preserved for Oil Red O staining, which is a sensitive test for microvesicular 
steatosis. Blood tests will hopefully soon be available for CK-18, a promising marker of apoptosis [40].

Beyond the bedside, regulatory agencies can assist in prevention and early detection by obtaining and performing long-term follow-up on patients from Phase 3 trials or patients with Phase 4 post-marketing exposure. This would allow prompt detection of long-term and often insidious drug-induced liver injury. Pharmaceutical companies can prevent release of such potentially dangerous drugs by proactively using better models for cellular testing of mitochondria toxicity when suspicions arise. Many techniques are currently available $[9,10]$. The use of better animal models that replicate the human disease phenotype $[44,64]$ could also unmask latent toxic potentials.

Rigorous application of the insights listed above could help anticipate and remedy potential problems. Therapeutic approaches could be implemented to arrest or reverse injury where possible. Examples include use of free radical scavengers, anti-apoptotic agents or agents that protect mitochondrial membrane integrity or function.

Another lesson from the troglitazone experience is that there are a few important distinctions seen with mitochondrial toxicity. It is not truly consistent with a rigid idiosyncratic toxicity paradigm. Synergy with other liver diseases can occur, as can synergy with drugs due to factors independent of P450 metabolism. Pathways of mitochondrial injury occur late and often possess the potential to cause significant chronic liver damage.

Mitochondrially targeted drugs have significant promise, especially in the fields of oncology, infectious disease and metabolism. Their safe use will rely on an understanding of their mechanism of action and on a recognition of the times and settings in which they may be dangerous or inappropriate. Thoughtful coordination of clinical trial data, cellular experimental data and translational research will allow us to enjoy their vast therapeutic potential while simultaneously minimising risk to our patients' safety. It is incumbent on all involved to facilitate free and transparent exchange of scientific and clinical data so that these problems can be rapidly identified and solved. It is essential to identify patients who are most at risk and who should therefore avoid such potentially toxic medication, while allowing other notat-risk patients to benefit from the potential therapeutic uses of such medications.

Acknowledgements We thank P. Rinschler and B. Rice for their help with editing. Thanks also to $\mathrm{M}$. Gerschenson (University of Hawaii Medical School, Honolulu, Oahu, HI, USA) for all her expert advice on mitochondrial pathophysiology.

Duality of interest N. L. Julie served as a medical expert in the troglitazone litigation from 2001 to 2005 in cases of his own and of numerous other patients. Otherwise the authors declare that there is no duality of interest associated with this manuscript.

\section{References}

1. Bjornsson E, Olsson R (2005) Suspected drug-induced liver fatalities reported to the WHO database. Dig Liver Dis 38:33-38

2. Peterson KF, Dufour S, Befroy D, Garcia R, Shulman G (2004) Impaired mitochondrial activity in the insulin-resistant offspring of patients with type 2 diabetes. N Engl J Med 350:664-671

3. Hawley J, Lessard SJ (2007) Mitochondrial function: use it or lose it. Diabetologia 50:699-702

4. Zimmerman HJ (1999) Hepatotoxicity the adverse effects of drugs and other chemicals on the liver. Lippincott Williams and Wilkins, Philadelphia, pp 122-123

5. Fromenty B, Robin MA, Igoudjil A, Mansouri A, Pessayre D (2004) The ins and outs of mitochondrial dysfunction in NASH. Diabetes Metab 30:121-138

6. Begriche K, Igoudjil A, Pessayre D, Fromenty B (2006) Mitochondrial dysfunction in NASH: causes, consequences and possible means to prevent it. Mitochondrion 6:1-28

7. Bailey SM, Pietsch EC, Cunningham CC (1999) Ethanol stimulates the production of reactive oxygen species at mitochondrial complexes I and III. Free Radic Biol Med 27:891-900

8. Cahill A, Cunningham CC, Adachi M et al (2002) Effects of alcohol and oxidative stress on liver pathology: the role of the mitochondrion. Alcohol Clin Exp Res 26:907-915

9. Lutsenko S, Cooper MJ (1998) Localization of the Wilson's disease protein product to mitochondria. Proc Natl Acad Sci U S A 95:6004-6009

10. Dykens J, Marroquin LD, Will Y (2007) Strategies to reduce latestage drug attrition due to mitochondrial toxicity. Expert Rev Mol Diagn 7:161-175

11. Amacher D (2005) Drug-associated mitochondrial toxicity and its detection. Curr Med Chem 12:1829-1839

12. Chang CC, Petrelli M, Tomashefski AF Jr et al (1999) Severe intrahepatic cholestasis caused by amiodarone toxicity after withdrawal of the drug: a case report and review of the literature. Arch Pathol Lab Med 123:251-256

13. Nadanaciva S, Dykens J, Bernal A, Capaldi RA, Will Y (2007) Mitochondrial impairment by PPAR agonists and statins identified via immunocaptured OXPHOS complex activities and respiration. Toxicol Appl Pharmacol 223:277-287

14. Masubuchi Y, Kano S, Horie T (2006) Mitochondrial permeability transition as a potential determinant of hepatotoxicity of antidiabetic thiazolidinediones. Toxicology 222:233-239

15. Shishido S, Koga H, Harada M et al (2003) Hydrogen peroxide overproduction in megamitochondria of troglitazone-treated human hepatocytes. Hepatology 37:136-147

16. Smith M (2003) Mechanisms of troglitazone hepatotoxicity. Chem Res Toxicol 16:679-687

17. Feinstein DL, Spagnolo A, Akar C et al (2005) Receptorindependent actions of PPAR thiazolidinedione agonists: is mitochondrial function the key? Biochem Pharmacol 70:177-188

18. Gitlin N, Julie N, Spurr C, Kie L, Juarbe H (1998) Two cases of severe clinical and histologic hepatotoxicity associated with troglitazone. Ann Intern Med 129:36-38

19. Booth AM, Caldwell SH, Iezzoni JC (2000) Troglitazoneassociated hepatic failure. Am J Gastroenterol 95:557-558

20. Hirose S, Kamoi K, Sasaki H (1998) A case of severe troglitazone-induced liver injury in a non-insulin-dependent diabetes mellitus patient. J Japan Diab Soc 41:295-299

21. Graham D, Green L (2000) Memorandum. Final report: Liver failure risk with troglitazone (Rezulin) NDA: 20-720. USDHHS, FDA, Center for Drug Evaluation and Research, Bethesda, MD

22. Jagannath S, Rai R (2000) Rapid-onset subfulminant liver failure associated with troglitazone. Ann Intern Med 132:677 
23. Bell D, Ovalle F (2000) Late-onset troglitazone-induced hepatic dysfunction. Diabetes Care 23:128-129

24. Caldwell S, Hespenheide EE, Von Borstel R (2001) Myositis, microvesicular hepatitis, and progression to cirrhosis from troglitazone added to simvastatin. Dig Dis Sci 46:376-378

25. Li H, Heller D, Leevy C, Zierer KG, Klein KM (2000) Troglitazone-induced fulminant hepatitis: report of a case with autopsy findings. J Diabetes Complicat 14:175-177

26. Herrine S, Choudhary C (1999) Severe hepatotoxicity associated with troglitazone. Ann Intern Med 130:163-164

27. Bissell D, Gores GJ, Laskin DL, Hoofnagle J (2001) Drug-induced liver injury: mechanisms and test systems. Hepatology 33:1009-1013

28. Chinnery P, DiMauro S (2005) Mitochondrial hepatopathies. J Hepatol 43:207-209 (Letter)

29. Gillis L, Sokol R (2003) Gastrointestinal manifestations of mitochondrial disease. Gastrointestinal Clin N Am 32:789-817

30. Pessayre D, Fromenty B, Mansouri A, Berson A (2003) Hepatotoxicity due to mitochondrial injury. Marcel Dekker, New York

31. Kleiner D, Gaffey MJ, Sallie R, Tsokos M et al (1997) Histopathologic changes associated with fialuridine hepatotoxicity. Mod Pathol 10:192-199

32. Toghill PJ, Smith PG, Benton P, Brown RC, Matthews HL (1974) Methyldopa liver damage. BMJ 3:545-548

33. Zimmerman HJ, Ishak K (1982) Valproate-induced hepatic injury: analyses of 23 fatal cases. Hepatology 2:591-597

34. Singhal A, Ghosh P, Khan SA (2003) Low dose amiodarone causing pseudo-alcoholic cirrhosis. Age Ageing 32:224-225

35. Snir Y, Pick N, Riesenberg K, Yanai-Inbar I, Zirkin H, Schlaeffer F (1995) Fatal hepatic failure due to prolonged amiodarone treatment. J Clin Gastroenterol 20:265-266

36. Rätz Bravo AE, Drewe J, Schlienger RG, Krähenbühl S, Pargger $\mathrm{H}$, Ummenhofer W (2005) Hepatotoxicity during rapid intravenous loading with amiodarone: Description of three cases and review of the literature. Crit Care Med 133:128-134

37. Naviaux RK, Nguyen K (2004) POLG mutations associated with Alpers' syndrome and mitochondrial DNA depletion. Ann Neurol 55:706-712

38. Berson A, De Beco V, Letteron P et al (1998) Steatohepatitisinducing drugs cause mitochondrial dysfunction and lipid peroxidation in rat hepatocytes. Gastroenterology 114:764-774

39. Pessayre D, Berson A, Fromenty B, Mansouri A (2001) Mitochondria in steatohepatitis. Semin Liver Dis 21:57-69

40. Wieckowska A, Zein N, Yerian L, Lopez A, McCullough A, Feldstein A (2006) In vivo assessment of liver cell apoptosis as a novel biomarker of disease severity in nonalcoholic fatty liver disease. Hepatology 44:27-33

41. Scatena R, Bottoni P, Martorana GE et al (2007) Mitochondria, ciglitazone and liver: a neglected interaction in biochemical pharmacology. Eur J Pharmacol 567:50-58

42. Masubuchi Y (2006) Metabolic and non-metabolic factors determining troglitazone hepatotoxicity: a review. Drug Metab Pharmacokinet 21:347-356

43. Haskins J, Rowse P, Rahbari R, de la Iglesia F (2001) Thiazolidinedione toxicity to isolated hepatocytes revealed by coherent multiprobe fluorescence microscopy and correlated with multiparameter flow cytometry of peripheral leukocytes. Arch Toxicol 75:425-438

44. Ong MM, Latchoumycandane C, Boelsterli UA (2007) Troglitazone-induced hepatic necrosis in an animal model of silent genetic mitochondrial abnormalities. Toxicol Sci 97:205-213

45. Bohan A, Boyer J (2002) Mechanisms of hepatic transport of drugs: implications for cholestatic drug reactions. Seminar in Liver Disease 22:123-136

46. Funk C, Ponelle C, Scheuermann G, Pantze M (2001) Cholestatic potential of troglitazone as a possible factor contributing to troglitazone-induced hepatotoxicity: in vivo and in vitro interaction at the canalicular bile salt export pump (Bsep) in the rat. Mol Pharmacol 59:627-635

47. Dhawan M, Agrawal R, Ravi J et al (2002) Rosiglitazone-induced granulomatous hepatitis. J Clin Gastroenterol 34:582-584

48. Bonkovsky HL, Azar R, Bird S, Szabo G, Banner B (2002) Severe cholestatic hepatitis caused by thiazolidinediones: risks associated with substituting rosiglitazone for troglitazone. Dig Dis Sci 47:1632-1637

49. Nagasaka S, Abe T, Kawakami A et al (2002) Pioglitazoneinduced hepatic injury in a patient previously receiving troglitazone with success. Diabet Med 19:344-348

50. Chase M, Yarze J (2002) Pioglitazone-associated fulminant hepatic failure. Am J Gastroenterol 97:502 (Letter)

51. Al-Salman J, Arjomand H, Kemp D, Mittal M (2000) Hepatocellular injury in a patient receiving rosiglitazone. Ann Intern Med 132:121-124

52. Pinto AG, Cummings O, Chalasani N (2002) Severe but reversible cholestatic liver injury after pioglitazone therapy. Ann Intern Med 137:857

53. Brunmair B, Staniek K, Gras F et al (2004) Thiazolidinediones, like metformin, inhibit respiratory complex I: a common mechanism contributing to their antidiabetic actions? Diabetes 53:1052-1059

54. Bedoucha M, Atzpodien E, Boelsterli UA (2001) Diabetic KKAy mice exhibit increased hepatic PPARgammal gene expression and develop hepatic steatosis upon chronic treatment with antidiabetic thiazolidinediones. J Hepatol 35:17-23

55. Colca JR, McDonald W, Waldon DJ et al (2004) Identification of a novel mitochondrial protein ("mitoNEET") cross-linked specifically by a thiazolidinedione photoprobe. Am J Physiol Endocrinol Metab 286:E252-E260

56. Alvarez-Sanchez R, Montavon F, Hartung T, Pähler A (2006) Thiazolidinedione bioactivation: a comparison of the bioactivation potentials of troglitazone, rosiglitazone, and pioglitazone using stable isotope-labeled analogues and liquid chromatography tandem mass spectrometry. Chem Res Toxicol 19:1106-1116

57. Alsheikh-Ali AA, Karas R (2004) Adverse events with concomitant use of simvastatin or atorvastatin and thiazolidinediones. Am J Cardiol 93:1417-1418

58. Ledl M, Hohenecker J, Francesconi C, Roots I, Bauer MF, Roden M (2005) Acute myopathy in a type 2 diabetic patient on combination therapy with metformin, fenofibrate and rosiglitazone. Diabetologia 48:1996-1998

59. Tafazoli S, Spehar D, O'Brien P (2005) Oxidative stress mediated idiosyncratic drug toxicity. Drug Metab Rev 37:311-325

60. Revuelta M (2004) Cumulative insults to mitochondrial function may promote the emergence of 'syndrome $\mathrm{X}$ ' and diabetes mellitus in HIV/HCV co-infected patients. Mitochondrion 4:175-184

61. Savès M, Raffi F, Clevenbergh $P$ et al (2000) Hepatitis B or hepatitis $\mathrm{C}$ virus infection is a risk factor for severe hepatic cytolysis after initiation of a protease inhibitor-containing antiretroviral regimen in human immunodeficiency virus-infected patients. The APROCO Study Group. Antimicrob Agents Chemother 44:3451-3455

62. den Brinker M, Wit FW, Wertheim-van Dillen PM et al (2000) Hepatitis B and C virus co-infection and the risk for hepatotoxicity of highly active antiretroviral therapy in HIV-1 infection. AIDS 14:2895-2902

63. Aceti A, Pasquazzi C, Zechini B, De Bac C, LIVERHAART Group (2002) Hepatotoxicity development during antiretroviral therapy containing protease inhibitors in patients with HIV: the role of hepatitis B and C virus infection. J Acquir Immune Defic Syndr 29:41-48

64. Boelsterli U (2003) Animal models of human disease in drug safety assessment. J Toxicol Sci 28:109-121 